\title{
Synbiotic Supplementation Modulates Gut Microbiota, Regulates Beta-Catenin Expression And Prevents Weight Gain in ob/ob Mice
}

\author{
Sebastião Mauro Bezerra Duarte \\ Universidade de São Paulo \\ José Tadeu Stefano \\ Universidade de São Paulo \\ Lucas A. M. Franco \\ Universidade de São Paulo \\ Roberta C. Martins \\ Universidade de São Paulo \\ Bruna D. G. C. Moraes \\ Universidade de São Paulo \\ Denise Frediani Barbeiro \\ Universidade de São Paulo \\ Nathalia Oliveira \\ Universidade de São Paulo \\ Junia Marielle Teixeira Rodrigues Neri \\ Universidade de São Paulo \\ Denise Siqueira Vanni \\ Universidade de São Paulo \\ Ester C. Sabino \\ Universidade de São Paulo \\ Flair José Carrilho \\ Universidade de São Paulo \\ Claudia P. Oliveira ( $\nabla$ cpm@usp.br) \\ Universidade de São Paulo
}

\section{Research Article}

Keywords: gut microbiota, synbiotic supplementation, probiotics, prebiotics, ob/ob mice

Posted Date: November 23rd, 2021

DOl: https://doi.org/10.21203/rs.3.rs-655169/v2 
License: (c) (i) This work is licensed under a Creative Commons Attribution 4.0 International License. Read Full License 


\section{Abstract}

Background: The aim of this study was to examine the impact of synbiotic supplementation in obesity and microbiota in ob/ob mice. 20 animals were divided into four groups: Obese Treated (OT), Control (OC), Lean Treated (LT) and Control (LC). All animals received standard diet for 8 weeks. Treated groups received a synbiotic in water while nontreated groups received water. After 8 weeks, all animals were sacrificed and gut tissue mRNA isolation and stool samples by microbiota analysis were collected. Betacatenin, occludin, cadherin and zonulin were analyzed in gut tissue by RT-qPCR.

Results: The synbiotic supplementation reduced body weight gain in OT comparing with OC $(p=0.0398)$, increase of Enterobacteriaceae $(p=0.005)$ and decrease of Cyanobacteria $(p=0.047)$, Clostridiaceae $(\mathrm{p}=0.026)$, Turicibacterales $(\mathrm{p}=0.005)$ and Coprococcus $(\mathrm{p}=0.047)$. A significant reduction of Sutterella bacteria $(p=0.009)$ and Turicibacter $(p=0.005)$ was observed in LT compared to LC. Alpha and beta diversities were differ between all treated groups. Beta-catenin gene expression was significantly decreased in the gut tissue of OT $(p \leq 0.0001)$ when compared to other groups. No changes were observed in occludin, cadherin and zonulin gene expression in the gut tissue.

Conclusion: The synbiotics supplementation prevents excessive weight gain, modulates the gut microbiota, and reduces beta-catenin expression in ob/ob mice.

\section{Introduction}

Some studies have associated obesity with gut microbiota (GM), small intestinal bacterial overgrowth (SIBO), permeability alteration as well the increase in lipopolysaccharide (LPS) production $[1,2]$. These processes induce metabolic endotoxemia, inflammation, impaired glucose metabolism, insulin resistance (IR), obesity, and contribute to the development of metabolic syndrome (MetS) and type 2 diabetes mellitus (T2DM) [3].

The term symbiotic refers to food ingredients or dietary supplements that combine probiotics and prebiotics in a form of synergism, for example, a mixtures of fructooligosaccharides (FOS) with lactisol Bifidobacteriase with Lactobacillus [4]. These promote the survival and implantation of probiotics in the large intestine. Some studies have shown that the consumption of symbiotics prevents bacterial translocation, epithelial invasion and inhibits bacterial adhesion of the mucosa and the production of antimicrobial peptides, reducing inflammation and stimulating host immunity $[5,6]$. Intestinal colonization of synbiotic bacteria seems to be a good strategy to reduce the damage caused by SIBO and gut permeability. Probiotics are live microbial food supplements that promote: the maintenance of mucosal integrity [7]; the GM balance; the increment of humoral and cellular immunity and the reduction of cholesterol and triglycerides (TG) [8, 9]. Prebiotics are derived from naturally occurring carbohydrates in some vegetables, which are not hydrolyzed by digestive enzymes and reach the large intestine to be digested by GM. This type of supplement works as an energy source to the growth of beneficial bacteria [7]. 
The leptin deficiency causing hyperphagia, excessive nutrient intake and reduced energy expenditure leading to the development of MetS, with visceral obesity, IR, mimetizing obesity as well in humans [10]. Due to this phenotype that easily facilitates the development of IR, T2DM and inflammatory response, these animals are used in several experimental studies $[11,12]$. Due to paucity and the lack of standardization in studies evaluating the supplementation of prebiotics, probiotics and synbiotics in obesity, we proposed a study using an experimental model of leptin deficient obese mice (ob/ ob mice) to test the effects of synbiotic supplementation in this experimental model to evaluate the possible modulation of obesity, gut microbiota, gut integrity, and permeability.

\section{Material And Methods \\ 2.1. Animals}

Twenty adults male ob/ ob mice were housed in a temperature, humidity-, and ventilation-controlled vivarium, with a 12-h light/ dark cycle. All procedures for animal experimentation followed the ethical guidelines of the Helsinki Declaration of 1975 (NIH Publication No. 85-23, revised 1996) and the Guidelines of Animal Experimentation from the Faculdade de Medicina, Universidade de São Paulo FMUSP.

This study was developed at the Laboratório de Gastroenterologia Clínica e Experimental LIM-07, Division of Clinical Gastroenterology and Hepatology, Hospital das Clínicas HCFMUSP, Department of Gastroenterology, FMUSP, Sao Paulo, SP, Brasil in collaboration with the Laboratório de Parasitologia LIM46 do Instituto de Medicina Tropical da FMUSP, the Laboratório de Emergências Clínicas LIM-51 da FMUSP.

\subsection{Experimental procedures}

The animals were divided into four groups: Obese Treated [OT $(n=5)]$, Obese Control [OC $(n=5)]$, Lean Treated [LT ( $n=5)$ ], Lean Control (lean mice) [LC ( $n=5)]$. The OT and LT groups received drinking water with a combination of probiotics and prebiotics [Lactobacillus acidophilus SD 5221 [109 colony forming unit (CFU)], Lactobacillus rhamnosus SD 5675 (109 CFU), Lactobacillus paracasei SD 5275 (10 $\mathrm{CFU})$, Bifidobacterium lactis SD $5674\left(10^{9} \mathrm{CFU}\right)$ e frutooligossacarídeo (5.5 g) (Simbioflora - Invictus Farmanutrição Ltda, Sao Paulo, Brazil) for eight weeks. The OC and LC groups received only potable water. For all groups was given standard diet Nuvilab CR1 (Nuvital Nutrientes S/A, Colombo, Brazil) (see Additional file 1).

The animal's weight was measured using a digital balance (Gehaka, Model BK4001, Brazil) and the weight gain was calculated as the difference between body weight measured at the beginning and at the end of the protocol.

After the period of treatment, the animals were anesthetized with ketamine hydrochloride $(0.1 \mathrm{~mL} / \mathrm{kg})$ intraperitoneally and sacrificed. Hepatic tissue samples were collected for histological analysis. Gut 
tissue samples were collected for analysis of the mRNAs of genes related to gut integrity [beta-catenin ( $5^{\prime}$ GTGCAATTCCTGAGCTGACA 3' - 5' CTTAAAGATGGCCAGCAAGC 3'), occludin (5' CCTCCAATGGCAAAGTGAAT 3' - 5' CTCCCCACCTGTCGTGTAGT 3'), cadherin (5' ACTGTGAAGGGACGGTCAAC 3' - 5' TGTCCCGGGTATCATCATCT 3') and zonulin (5' CCACCTCTGTCCAGCTCTTC 3' - 5' CACCGGAGTGATGGTTTTCT 3'). The gene expression was evaluated by Real time quantitative reverse transcription polymerase chain reaction (RT-qPCR). To control the RTqPCR reactions, internal control Beta-2 microglobulin ( $\beta 2 \mathrm{M}$ ) (5' CCAGCGUACUCCAAAGAUUTT 3' - 5' AAUCUUUGGAGUACGCUGGTT 3') gene was used.

\subsection{Gene expression}

\subsubsection{Extraction of RNA from gut tissue}

For extraction, the gut tissue was fragmented into a tissue sprayer (Micro-Dismenbrenator II B. Braun Biotech International, Melsugen, Germany). To the pulverized material, $1 \mathrm{~mL}$ TRIzol® (Invitrogen Life Technologies, Carlsbad, CA, USA) was added and the solution incubated for $5 \mathrm{~min}$ at room temperature. To the solution, $200 \mu \mathrm{L}$ of chloroform (Merck, Darmstadt, Germany) was added and it was vigorously stirred for $15 \mathrm{sec}$, followed by incubation for $5 \mathrm{~min}$ at room temperature and centrifugation for $15 \mathrm{~min}$, $12.000 \mathrm{rpm}$ at $4^{\circ} \mathrm{C}$ (Eppendorf 5417-R, Hamburg, Germany). The supernatant was then transferred to a new $1.5 \mathrm{~mL}$ sterile tube and RNA was precipitated with $500 \mu \mathrm{L}$ of isopropanol (Merck, Darmstadt, Germany).

The mixture should stand for $10 \mathrm{~min}$ and centrifuged for $15 \mathrm{~min}, 12.000 \mathrm{rpm}$ at $4^{\circ} \mathrm{C}$ (Eppendorf $5417-\mathrm{R}$, Hamburg, Germany). The supernatant was removed, and the RNA bud washed with $1 \mathrm{~mL}$ of $70 \%$ ethanol (Merck, Darmstadt, Germany), was centrifuged again and resuspended in an appropriate volume of sterile water [(UltraPure ${ }^{\mathrm{TM}}$ DNase/ RNase-Free Distilled Water (Invitrogen Life Technologies, Carlsbad, CA, USA)].

\subsubsection{Quantification and Analysis of Total RNA Integrity}

The concentration of total RNAs extracted was determined by spectrophotometry [NanoDrop ND-1000 (NanoDrop Technologies, Wilmington, DE, USA)]. RNA preparation was considered protein free when the A260/280 ratio was between 1.8 and 2.0. For samples that did not reach these values, purifications were performed using the RNeasy ${ }^{\text {TM }}$ Mini Kit (Qiagen, Hilden, Germany). The integrity and purity of the RNAs were analyzed by $1 \%$ agarose gel electrophoresis. Only samples whose bands corresponding to ribosomal RNA (RNA) 18 and 28S were shown to be intact for analysis under ultraviolet light. The RNAs were maintained at $-80^{\circ} \mathrm{C}$ until use.

\subsubsection{Real-time PCR}

One hundred nanograms were used for Real-time PCR analysis. PCR was performed in a $15 \mu \mathrm{L}$ reaction mixture containing 7.5 $\mu \mathrm{L} 2 \times$ SYBR Green Reaction Mix (Invitrogen Life Technologies, Carlsbad, CA, USA), $0.3 \mu \mathrm{L}$ each primer (10 pmol), $0.3 \mu \mathrm{L}$ Super Script III RT/Platinum Taq Mix (10 pmol/ $\mu \mathrm{L}), 0.15 \mu \mathrm{L}$ ROX 
Reference Dye, and $5 \mu \mathrm{L}$ sample in water. Gene-specific primers were used. Reactions were performed using StepOne ${ }^{\mathrm{TM}}$ Real-Time PCR System (Applied Biosystem - Foster City, CA - EUA).

\subsubsection{Analysis of Fecal microbiota}

\subsubsection{Fecal samples collection, DNA extraction and sequencing}

The stool samples $(n=20)$ collected were mixed with RNAlater (Life Technologies Corporation, Carlsbad, $\mathrm{CA}$ ) and stored at $-20^{\circ} \mathrm{C}$. After the maximum period of four hours, they were separated into aliquots of $200 \mathrm{mg}$ each and stored at $-80^{\circ} \mathrm{C}$. We used 0.25 grams of each stool sample for analyses.

Approximately $0.25 \mathrm{~g}$ of feces was used for DNA isolation using the DNeasy PowerSoil Kit (Qiagen, Germantown, MD, USA), following the manufacturer's instructions. The extracted DNA was quantified by the Qubit ${ }^{\circledR} 4.0$ fluorometer using the dsDNA HS Assay kit (Invitrogen ${ }^{\mathrm{T}}$, CA, USA) according to the manual. PCR amplification was then carried out using primers for the $V 4$ region of the 16S rRNA gene [bacterial/archaeal primer set - 515F (5'-CACGGTCGKCGGCGCCATT-3')/ 806R (5'GGACTACHVGGGTWTCTAAT-3 [13]. The amplification was confirmed by electrophoresis in a $1.5 \%$ agarose gel with 1 X TAE buffer. The template preparation was performed by the lon Chef System (Thermo Fisher Scientific, MA, USA), using the Ion PGM Hi-Q View Chef Kit. Sequencing was performed in the Ion Personal Genome Machine (PGM), using the lon PGM Hi-Q Sequencing Kit and the lon 318 Chip v2, following the instructions of the manufacturer (Thermo Fisher Scientific, MA, USA). Samples beneath 85.000 reads were re-sequenced.

\subsubsection{Analysis of results}

Data analysis were processed through the Quantitative Insights Into Microbial Ecology (QIIME) software package v1.8 [14]. Reads were filtered by length $(>200$ pb), quality (Phred Score $=30)$ and minimum expected error (0.1) utilizing the USEARCH tool [15]. The remaining sequences were grouped into Operational Taxonomic Units (OTUs), based on 97\% similarity, using the UCLUST algorithm. Singletons were removed. OTUs were classified taxonomically, using the Greengenes 16S reference database v. 13.8 [16]. Alpha-diversity index (Shannon, Simpson, Chao1, Observed_OTUs, Faith's Phylogenetic diversity and Pielou's Evenness) and Beta diversity index (Weighted Unifrac, Unweighted Unifrac, Bray Curtis and Jaccard) were calculated based on the rarefied OTU table using 54789 sequences per sample. The Principal Coordinates Analysis (PCoA) plot for each of the beta diversity index was generated using Emperor [17]. Compositions of microbiota communities were summarized by proportion at different taxonomy levels, including genus, family, order, class, and phylum ranks. The Kruskal-Wallis test was performed to explore differences in alpha diversity index. Differences in community composition (beta diversity) were assessed using Permutational Multivariate Analysis of Variance (PERMANOVA). KruskalWallis and PERMANOVA analysis were corrected by Benjamini \& Hochberg method. 
Microorganism features distinguishing fecal microbiota were identified using the linear discriminant analysis (LDA) effect size (LEfSe) method for biomarker discovery, which emphasizes both statistical significance and biological relevance (metagenomic biomarker discovery and explanation). LEfSe uses the Kruskal-Wallis rank-sum test with a normalized relative abundance matrix to detect features with significantly different abundances between assigned taxa and performs LDA to estimate the effect size of each feature.

\subsection{Statistical Analysis}

All data were expressed as mean or median (depending on the distribution pattern of the variables). Minimum (Min), Maximum (Max) and Standard Deviation (DP) values were set. For Gaussian distribution variables we used t-test, one-way ANOVA and the Newman-Keuls post-test of multiple comparisons. For non-Gaussian distribution variables, the Mann-Whitney, Kruskal-Wallis and Dunn's multiple-comparison tests were used. Chi-square tests and Fisher's exact test were used to compare the histological scores between the groups. A $p$ value $<0.05$ was considered significant. All calculations and graphs were performed with SPSS 17.0 software (SPSS Inc., Chicago, IL, USA).

\section{Results}

\subsection{Weighing of animals}

The body weight gain after synbiotic supplementation in the OT group was significantly lower when compared with OC group $(p=0.0398)$. No relevant modifications were observed in the body weight gain when it was compared LT and LC groups (Figure 1).

\subsection{Analysis of intestinal microbiota}

Microorganisms belonging to the bacteria kingdom were searched and quantified following the taxonomic classification of phyla, class, order, family and genus (species subdivision was not contemplated in the analysis).

Rarefaction curves showed that the number of observed OTUs plateaued after 54789 reads, suggesting that we have a good representation of the microbial community. Significant differences were observed in the evaluated alpha diversity indexes. [Chao1: LC vs OT $(p=0.009)$, LC vs OC $(p=0.028), L T$ vs OC $(p=$ $0.009)$, LT vs OT $(\mathrm{p}=0.009)$ and OC vs OT $(\mathrm{p}=0.009)$ groups. No significance difference was observed between LC vs LT groups ( $\mathrm{p}=0.75)$ (Figure 2a). Observed OTUs: LC vs OT ( $\mathrm{p}=0.009)$, LT vs OT ( $\mathrm{p}=$ $0.009)$ and OC vs OT $(\mathrm{p}=0.016)$ groups. No significance difference was observed between LT vs OC $(\mathrm{p}=$ $0.075)$, LC vs LT $(\mathrm{p}=0.83)$ and LC vs OC $(\mathrm{p}=0.17)$ groups (Figure $2 \mathrm{~b})$. Faith's Phylogenetic diversity. LC vs OC ( $\mathrm{p}=0.047)$, LC vs OT ( $\mathrm{p}=0.009)$, LT vs OC $(\mathrm{p}=0.028)$, LT vs OT $(\mathrm{p}=0.009)$ and OC vs OT ( $\mathrm{p}=$ $0.016)$ groups. No significance difference was observed between LC $v s$ LT groups $(p=0.91)$ groups (Figure 2c)]. On the hand, no significance differences were observed in the alpha diversity indexes (Shannon, Simpson and Pielou's Evenness) between the groups. 
The plots revealed that samples were clustered according to their bacterial composition. PERMANOVA indicated there was a significant difference in beta diversity between the groups [Bray Curtis: LC vs LT ( $\mathrm{p}$ $=0.012), \mathrm{LC} v s \mathrm{OC}(\mathrm{p}=0.010), \mathrm{LC}$ vs OT $(\mathrm{p}=0.008), \mathrm{LT}$ vs OC $(\mathrm{p}=0.009)$ and LT vs OT $(\mathrm{p}=0.008)$ groups. No significance difference was observed between OC vs OT groups $(\mathrm{p}=0.111)$ groups (Figure 3a). Unweighted Unifrac: LC vs LT groups $(\mathrm{p}=0.012)$, LC vs OC $(\mathrm{p}=0.010), \mathrm{LC}$ vs OT $(\mathrm{p}=0.008), \mathrm{LT}$ vs OC $(p=0.009)$ and LT vs OT $(p=0.008)$ groups. No significance difference was observed between OC vs OT groups $(p=0.111)$ groups (Figure $3 b)$. Weighted Unifrac: LT vs OC $(p=0.008)$ and LT vs OT $(p=0.019)$. No significance difference was observed between LC vs LT $(\mathrm{p}=0.15)$, LC vs OC $(\mathrm{p}=0.209)$, LC vs OT $(\mathrm{p}=$ $0.078)$ and OC vs OT ( $(\mathrm{p}=0.61)$ groups (Figure 3c). Jaccard: LC $v s$ LT $(\mathrm{p}=0.009)$, LC vs OC $(\mathrm{p}=0.011), \mathrm{LC}$ vs OT $(\mathrm{p}=0.012)$, LT vs OC $(\mathrm{p}=0.008)$, LT vs OT $(\mathrm{p}=0.006)$ and OC vs OT $(\mathrm{p}=0.009)$ groups (Figure 3d)].

The metagenome analysis LEfSe approach was applied to identify the key phylotypes responsible for the difference between the groups. Bacteroides and Bacteroidaceae, which were most abundant in the treated groups (lean and obese) where compared to controls, were the dominant phylotypes that contributed to the difference between the intestinal microbiota of treated groups (Figure $4 a$ and $b$ ).

Regarding the phyla the proportion of Bacteroidetes and Firmicutes observed among samples in the OT group compared to the $\mathrm{OC}$ was $71.02 \%$ and $18.19 \%$, respectively (Figure $5 a$ ). Figure $5 \mathrm{~b}$ shows the heatmap representation calculated at the genus level of the classified reads obtained. The Cyanobacteria phylum and the Turicibacterales order were reduced in OT group ( $p=0.047, p=0.005$ respectively) and among bacteria classes there was an increase in the Gammaproteobacteria $(p=0.005)$, in the bacteria order Enterobacteriales ( $p=0.005)$ and in the bacteria family Enterobacteriaceae $(p=0.005)$. In addition, the Clostridiaceae family $(p=0.026)$ and the bacteria genus Turicibacter $(p=0.005)$ and Coprococcus $(p$ $=0.047$ ) were decreased in OT group (Figure 6).

Analyzing the samples of lean mice, we observed that in the LT group when compared to the LC there was an increase in the: bacteria of the Tenericutes phylum ( $p=0.028)$; Enterobacteriales order $(p=0.005)$; Bacteroidaceae $(p=0.028)$; Prevotellaceae $(p=0.028)$; Enterobacteriaceae family $(p=0.005)$; Bacteroides genus ( $p=0.028)$ and Lactococcus $(p=0.019)$ genus. There was also a decrease in the Turicibactergenus $(p=0.005)$ and Sutterella genus ( $p=0.009)$ in LT group (Figure 7).

\subsection{Gene expression analysis}

A significantly decrease in gene expression of beta-catenin in the gut tissue was observed in OT group when compared to OC group $(p=0.0479)$ and in LT group when compared to LC group $(p=0.0030)$ (Figure 8). On the other hand, no significant changes were verified among the groups in gene expression of cadherin $(p=0.4048)$, occludin $(p=0.2063)$ and zonulin $(Z 0-1)(p=0.171)$ in gut tissue (see Additional file 2).

\section{Discussion}


The present study demonstrated that dietary supplementation of synbiotics prevented excessive weigh gain in obese mice when compared to controls, modulated the GM and reduced the gene expression of beta-catenin expression. The last mentioned is an important gene involved in tight junction signaling, inflammation and obesity [18].

The mechanism by which synbiotic supplementation could influence the loss of weight has not been fully elucidated. However, corroborating our results, studies have demonstrated that dietary supplementation with probiotics and prebiotics combination is able to modulate the GM of mice and obesity humans, which leads to significant changes in the prevalence of specific intestinal bacteria. These population with dietary supplementation may benefit from the decrease of energy harvest capacity from diet and thus reduce weight gain $[19,20]$.

In the present study the intragroups analyses of alpha and beta diversities were differ between all treated groups and control group. Addicionaly, the intergroups analyses also showed a significant difference between lean and obese treated and control groups. Our data demosntrate that the richness and evenness of the GM in lean and obese animals were different before treatment and this difference became more evident after synbiotic supplementation. Corroboring our findings, studies with probiotics supplementation in human and mice may change GM richness and diversity for conditions such as obesity and metabolic disorders [21, 22]. Lower diversity in the GM has been linked to obesity, higher IR, higher visceral fat and numerous inflammatory conditions [23]. Thus, GM diversity could be linked to body weight.

The gut microbiota is mainly composed by bacteria from the Bacteroidetes and Firmicutes phylum. Proteobacteria, Verrucomicrobia, Actinobacteria, Fusobacteria, and Cyanobacteria are present in minor proportions [24]. Adequate amounts of Cyanobacteria are considered beneficial for the host to diminish inflammation through NF-KB inhibition, and, consequently, reduce proinflammatory cytokines, protecting the host against oxidative stress [25]. However, increased Cyanobacteria have been associated with obesity [26] and recent study published by Shao et al. showed that after weight loss the abundance of these bacteria decreases [27]. Our findings showed Cyanobacteria phylum reduction in the OT group after supplementation associated with weight loss, similarly to Shao et al [27].

Turicibacter is a genus in the Firmicutes phylum of bacteria that has been found most commonly in the gut [28]. Although this bacteria has been related to greater energy extraction from the diet and that would be related to obesity [1], the data on the literature are conflicting. There are studies that also show a negative correlation between the amount of Turicibacter and NF-KB and associate a lower amount of this bacteria with the most inflamed individuals with obesity and steatosis [3]. In our study, the analyzes of fecal microbiota of ob/ob mice treated (obese and lean) after synbiotics supplementation demonstrated a decrease of Turicibacter genus when compared to control groups, which could be a interesting finding, responsible for reducing the energy extraction from the diet. Besides, we observed an increase of Enterobacteriaceae family in relation to the control groups. This family is a group of bacteria considered 
beneficial either by promoting protection of the intestinal barrier or regulating the growth of other bacteria that promote gastrointestinal disorders [29].

The other relevant finding of our study was the decrease in Clostridiaceae family, and Coprococcus genus in the OT group and a reduction in the abundance of Sutterella genus in LT group. Clostridiaceae family is the group of bacteria present mainly in obese and in T2DM animals [30] and is associated with dysbiosis in adults and children [31]) and inflammatory bowel disease in adults [32]. In consonance with our findings, a recent study demonstrated that probiotic supplementation (Lactobacillus paracasei) is able to reduce the abundance of Clostridiaceae [33]. On the other hand, Coprococcus, a genus in the Firmicutes phylum, when increased has been associated with a high-fat diet in mice [34]. Bacteria of the genus Sutterella have been often associated with inflammatory bowel disease and disrupt of the intestinal epithelial homeostasis [35]. It is evident that the consumption of high-protein and high-sugar diets increase Sutterella in the gut [36] and that probiotics supplementation reduces their abundance [37], which corroborates our findings in the LT group.

In our study, a significant increase in the following groups of gut bacteria was observed in the LT group: Bacteroides and Lactococcus genus, Enterobacteriales order, Bacteroidaceae, Prevotelaceae and Enterobacteriaceae family. The abundance of some of these bacteria is linked to the improvement of the integrity of the intestinal barrier [38].

The main role of the intestinal barrier is to separate the internal environment from the luminal content, and the complex system of intercellular junctions, including tight junctions, seals together the epithelial cells to form a continuous layer [39]. In our study we observed a lower expression of intestinal betacatenin in OT and LT groups compared to control groups. There was no difference in cadherin, occludin and ZO-1. The beta-catenin is one of the proteins that compose the tight junctions which are primarily responsible for maintenance of the intestinal permeability barrier, regulating the passage of ions and solutes between cells by the paracellular pathway [40]. However, when beta-catenin expression is increased it would enter the cell nucleus and would induce the activation of NF-kB, pro-inflammatory genes and the expression of others oncogenes, which are important for development of some intestinal diseases [41, 42] and hepatocellular carcinoma (HCC) [43]. Evidence about the effect of synbiotic supplementation on beta-catenin modulation is scarce. Our results are consistent with Kuugbee et al. that has shown an inhibition of beta-catenin signaling pathway after probiotic [44] and synbiotic supplementation [18]. Based on these results we can infer that the reduction in beta-catenin expression improves the permeability of the intestinal barrier, preventing the passage of endotoxins from the intestinal lumen through the intestinal barrier and consequently not triggering inflammatory cytokines, which are important for the development of obesity. On the other hand, there are studies showing increased expression of tight junction after synbiotic administration, highlighting ZO-1, occludin and claudin, but not evaluating beta-catenin $[19,45]$. Apparently, modulation of intestinal tight junctions happens with prolonged use of symbiotic, which perhaps justifies no difference in gene expression of cadherin, occludin and ZO-1 in our study. 
There were strengths and limitations in our study that should be considered. The strengths of our study were the combination of four different probiotics strains, the choice of isogenic mice, the microbial sequencing techniques and the rigorous evaluation of gene expression and liver histology performed by a specialist. However, our study also had some limitations. First, we only chose one probiotic fiber to include in the synbiotic supplementation and the treatment period has lasted only 8 weeks. Perhaps for these reasons, we have not observed more consistent results in gene expression in these animals. Despite these limitations, we believe that our results are encouraging and support the consideration of larger, welldesigned studies to evaluate synbiotics supplementation as obesity prevention.

In conclusion, our experimental study with animal model shows that synbiotics supplementation is effective to prevent excessive weigh gain, positively modulates the gut microbiota, reduces beta-catenin expression, but was not able to improve other tight junctions gene expressions. Our data support the evidence of beneficial effects of synbiotics supplementation on prevention of obesity. Nonetheless, more randomized controlled trials are needed.

\section{Abbreviations}

B2M: Beta-2 microglobulin; CFU: Colony forming unit; DNA: Deoxyribonucleic acid; FOS:

Fructooligosaccharides; HCC: Hepatocellular carcinoma; GM: Gut microbiota; IR: Insulin resistance; LC: Lean Control; LPS: Lipopolysaccharides; MetS: Metabolic syndrome; NAFLD: Nonalcoholic fatty liver disease; NF-kß: Transcription factor kappa B; OC: Obese Control; OT: Obese Treated; OTUs: Operational taxonomic unit; PCR: Polymerase chain reaction; RNA: Ribonucleic acid; RT-qPCR: Polymerase Chain Reaction after Relative Quantitative Reverse Transcription; rRNA: Ribosomal RNAs; SIBO: Small intestinal bacterial overgrowth; TG: Triglycerides; LT: Lean Treated; T2DM: Type 2 diabetes melittus.

\section{Declarations}

\section{Acknowledgments}

Not applicable.

\section{Authors' contributions}

SMBD, JTS and CPO contributed to the acquisition, analyses, interpretation of experimental data, prepared figures, and wrote the manuscript. LAMF, RCM, BDGCM and ECS performed sample extraction, coordinated amplicon sequencing, and performed associated bioinformatics. DFB performance to gene expression analysis. NO, JMRN and DSV contributed to the acquisition of experimental data. FJC and $\mathrm{CPO}$ contributed to the design and critical review of the manuscript. All authors edited the manuscript and approved the final draft.

\section{Funding}


Part of this study was supported by the Coordenação de Aperfeiçoamento de Pessoal de Nível Superior Brasil (CAPES). The authors declare no conflict of interest.

\section{Ethics approval}

The study was approved by the Comissão de Ética no Uso de Animais (CEUA) of the FMUSP (process number 127/15) and is reported in accordance with Animal Research: Reporting of In Vivo Experiments (ARRIVE) guidelines (https://arriveguidelines.org).

\section{Consent for publication}

Not applicable

\section{Competing interests}

The authors declare that they have no competing interests.

\section{Author details}

${ }^{1}$ Laboratório de Gastroenterologia Clínica e Experimental LIM-07, Division of Clinical Gastroenterology and Hepatology, Hospital das Clínicas HCFMUSP, Department of Gastroenterology, Faculdade de Medicina, Universidade de Sao Paulo, Sao Paulo, SP, Brazil

${ }^{2}$ Department of Infectious Diseases, Instituto de Medicina Tropical, Faculdade de Medicina, Universidade de Sao Paulo, Sao Paulo, SP, Brazil

${ }^{3}$ Laboratório de Investigação Médica LIM-51, Faculdade de Medicina, Universidade de Sao Paulo FMUSP, São Paulo, SP, Brazil.

\section{References}

1. Jiao, N. et al. Gut microbiome may contribute to insulin resistance and systemic inflammation in obese rodents: a meta-analysis. Physiol Genomics, 50, 244-254 (2018).

2. Madrid, A. M., Poniachik, J., Quera, R. \& Defilippi, C. Small intestinal clustered contractions and bacterial overgrowth: a frequent finding in obese patients. Dig Dis Sci, 56, 155-160 (2011).

3. Halmos, T. \& Suba, I. [Physiological patterns of intestinal microbiota. The role of dysbacteriosis in obesity, insulin resistance, diabetes and metabolic syndrome]. Orv Hetil, 157, 13-22 (2016).

4. Schrezenmeir, J. \& de Vrese, M. Probiotics, prebiotics, and synbiotics-approaching a definition.Am J Clin Nutr2001; 73:361S-364S. https://doi.org/10.1093/ajcn/73.2.361s

5. Cesaro, C. et al. Gut microbiota and probiotics in chronic liver diseases. Dig Liver Dis, 43, 431-438 (2011). 
6. Sanders, M. E., Merenstein, D. J., Reid, G., Gibson, G. R. \& Rastall, R. A. Probiotics and prebiotics in intestinal health and disease: from biology to the clinic.Nat Rev Gastroenterol Hepatol2019. https://doi.org/10.1038/s41575-019-0173-3.

7. Jayakumar, S. \& Loomba, R. Review article: emerging role of the gut microbiome in the progression of nonalcoholic fatty liver disease and potential therapeutic implications. Aliment Pharmacol Ther, 50, 144-158 (2019).

8. Ipsen, D. H., Lykkesfeldt, J. \& Tveden-Nyborg, P. Molecular mechanisms of hepatic lipid accumulation in non-alcoholic fatty liver disease. Cell Mol Life Sci, 75, 3313-3327 (2018).

9. Xie, C. \& Halegoua-DeMarzio, D. Role of Probiotics in Non-alcoholic Fatty Liver Disease: Does Gut Microbiota Matter? Nutrients 2019;11. https://doi.org/10.3390/nu11112837

10. Anstee, Q. M. \& Goldin, R. D. Mouse models in non-alcoholic fatty liver disease and steatohepatitis research. Int J Exp Pathol, 87, 1-16 (2006).

11. Pelletier, R. M., Layeghkhavidaki, H. \& Vitale, M. L. Glucose, insulin, insulin receptor subunits a and $\beta$ in normal and spontaneously diabetic and obese ob/ob and $\mathrm{db} / \mathrm{db}$ infertile mouse testis and hypophysis. Reprod Biol Endocrinol, 18, 25 (2020).

12. Ahmad, A. et al. Adiponectin homolog novel osmotin protects obesity/diabetes-induced NAFLD by upregulating AdipoRs/PPARa signaling in ob/ob and db/db transgenic mouse models. Metabolism, 90, 31-43 (2019).

13. Caporaso, J. G. et al. Global patterns of $16 \mathrm{~S}$ rRNA diversity at a depth of millions of sequences per sample. Proc Natl Acad Sci U S A, 108 (Suppl 1), 4516-4522 (2011).

14. Kuczynski, J. et al. Using QIIME to analyze 16S rRNA gene sequences from microbial communities.Curr Protoc Microbiol2012;Chapter 1:Unit 1E.5. https://doi.org/10.1002/9780471729259.mc01e05s27

15. Edgar, R. C. Search and clustering orders of magnitude faster than BLAST., 26, 2460-2461 (2010).

16. DeSantis, T. Z. et al. Greengenes, a chimera-checked 16S rRNA gene database and workbench compatible with ARB. Appl Environ Microbiol, 72, 5069-5072 (2006).

17. Vázquez-Baeza, Y., Pirrung, M., Gonzalez, A. \& Knight, R. EMPeror: a tool for visualizing highthroughput microbial community data. Gigascience, 2, 16 (2013).

18. AM, S., Yasser, M. H. R. \& HO, G. A. A. KM. Modulation of JNK-1/ $\beta$-catenin signaling by Lactobacillus casei, inulin and their combination in 1,2-dimethylhydrazine-induced colon cancer in mice (Royal Society of Chemistry, 2019).

19. Shinde, T. et al. Synbiotic supplementation with prebiotic green banana resistant starch and probiotic Bacillus coagulans spores ameliorates gut inflammation in mouse model of inflammatory bowel diseases.Eur J Nutr2020. https://doi.org/10.1007/s00394-020-02200-9.

20. Vallianou, N. et al. Prebiotics, Synbiotics, Postbiotics, and Obesity: Current Evidence, Controversies, and Perspectives. Curr Obes Rep, 9, 179-192 (2020).

21. Cotillard, A. et al. Dietary intervention impact on gut microbial gene richness., 500, 585-588 (2013). 
22. Everard, A. et al. Microbiome of prebiotic-treated mice reveals novel targets involved in host response during obesity. ISME J, 8, 2116-2130 (2014).

23. Le Chatelier, E. et al. Richness of human gut microbiome correlates with metabolic markers., 5000 , 541-546 (2013).

24. Sekirov, I., Russell, S. L., Antunes, L. C. \& Finlay, B. B. Gut microbiota in health and disease. Physiol Rev, 90, 859-904 (2010).

25. Ku, C. S., Yang, Y., Park, Y. \& Lee, J. Health benefits of blue-green algae: prevention of cardiovascular disease and nonalcoholic fatty liver disease. J Med Food, 16, 103-111 (2013).

26. Kaplan, R. C. et al. Gut microbiome composition in the Hispanic Community Health Study/Study of Latinos is shaped by geographic relocation, environmental factors, and obesity. Genome Biol, 20, 219 (2019).

27. Shao, Y. et al. Alterations of Gut Microbiota After Roux-en-Y Gastric Bypass and Sleeve Gastrectomy in Sprague-Dawley Rats. Obes Surg, 27, 295-302 (2017).

28. Auchtung, T. A. et al. Complete Genome Sequence of Turicibacter sp. Strain H121, Isolated from the Feces of a Contaminated Germ-Free Mouse.Genome Announc2016;4. https://doi.org/10.1128/genomeA.00114-16.

29. Wang, L. \& Wan, Y-J-Y. The role of gut microbiota in liver disease development and treatment. 2019. p.3-18. https://doi.org/10.1016/j.livres.2019.02.001

30. Horie, M. et al. Comparative analysis of the intestinal flora in type 2 diabetes and nondiabetic mice. Exp Anim, 66, 405-416 (2017).

31. Goldenberg, J. Z. et al. Probiotics for the prevention of Clostridium difficile-associated diarrhea in adults and children. Cochrane Database Syst Rev, 12, CD006095 (2017).

32. Muñiz Pedrogo, D. A. et al. An Increased Abundance of Clostridiaceae Characterizes Arthritis in Inflammatory Bowel Disease and Rheumatoid Arthritis: A Cross-sectional Study. Inflamm Bowel Dis, 25, 902-913 (2019).

33. Ferrario, C. et al. Modulation of fecal Clostridiales bacteria and butyrate by probiotic intervention with Lactobacillus paracasei DG varies among healthy adults. J Nutr, 144, 1787-1796 (2014).

34. Velázquez, K. T. et al. Prolonged high-fat-diet feeding promotes non-alcoholic fatty liver disease and alters gut microbiota in mice. World J Hepatol, 11, 619-637 (2019).

35. Da Silva, H. E. et al. Nonalcoholic fatty liver disease is associated with dysbiosis independent of body mass index and insulin resistance. Sci Rep, 8, 1466 (2018).

36. Hiippala, K., Kainulainen, V., Kalliomäki, M., Arkkila, P. \& Satokari, R. Mucosal Prevalence and Interactions with the Epithelium Indicate Commensalism of. Front Microbiol, 7, 1706 (2016).

37. Liu, J. P. et al. Effects of different diets on intestinal microbiota and nonalcoholic fatty liver disease development. World J Gastroenterol, 22, 7353-7364 (2016).

38. Shimizu, H. \& Benno, Y. Membrane filter method to study the effects of Lactobacillus acidophilus and Bifidobacterium longum on fecal microbiota. Microbiol Immunol, 59, 643-652 (2015). 
39. Valdes, A. M., Walter, J., Segal, E. \& Spector, T. D. Role of the gut microbiota in nutrition and health. $B M J, 361, \mathrm{k} 2179$ (2018).

40. Campbell, H. K., Maiers, J. L. \& DeMali, K. A. Interplay between tight junctions \& adherens junctions. Exp Cell Res, 358, 39-44 (2017).

41. Niessen, C. M. Tight junctions/adherens junctions: basic structure and function. J Invest Dermatol, 127, 2525-2532 (2007).

42. Basu, S., Cheriyamundath, S. \& Ben-Ze'ev, A. Cell-cell adhesion: linking Wnt/ $\beta$-catenin signaling with partial EMT and stemness traits in tumorigenesis. F1000Res 2018;7.

https://doi.org/10.12688/f1000research.15782.1

43. Zhou, Z., Chen, J., Yao, H., Hu, H. \& Cancer, C.Front Oncol2018; 8:371.

https://doi.org/10.3389/fonc.2018.00371.

44. Tao, J. et al. Activation of $\beta$-catenin and Yap1 in human hepatoblastoma and induction of hepatocarcinogenesis in mice., 147, 690-701 (2014).

45. Kuugbee, E. D. et al. Structural Change in Microbiota by a Probiotic Cocktail Enhances the Gut Barrier and Reduces Cancer via TLR2 Signaling in a Rat Model of Colon Cancer. Dig Dis Sci, 61, 2908-2920 (2016).

46. Yao, F. et al. Effect of Lactobacillus paracasei N1115 and fructooligosaccharides in nonalcoholic fatty liver disease. Arch Med Sci, 15, 1336-1344 (2019).

\section{Figures}
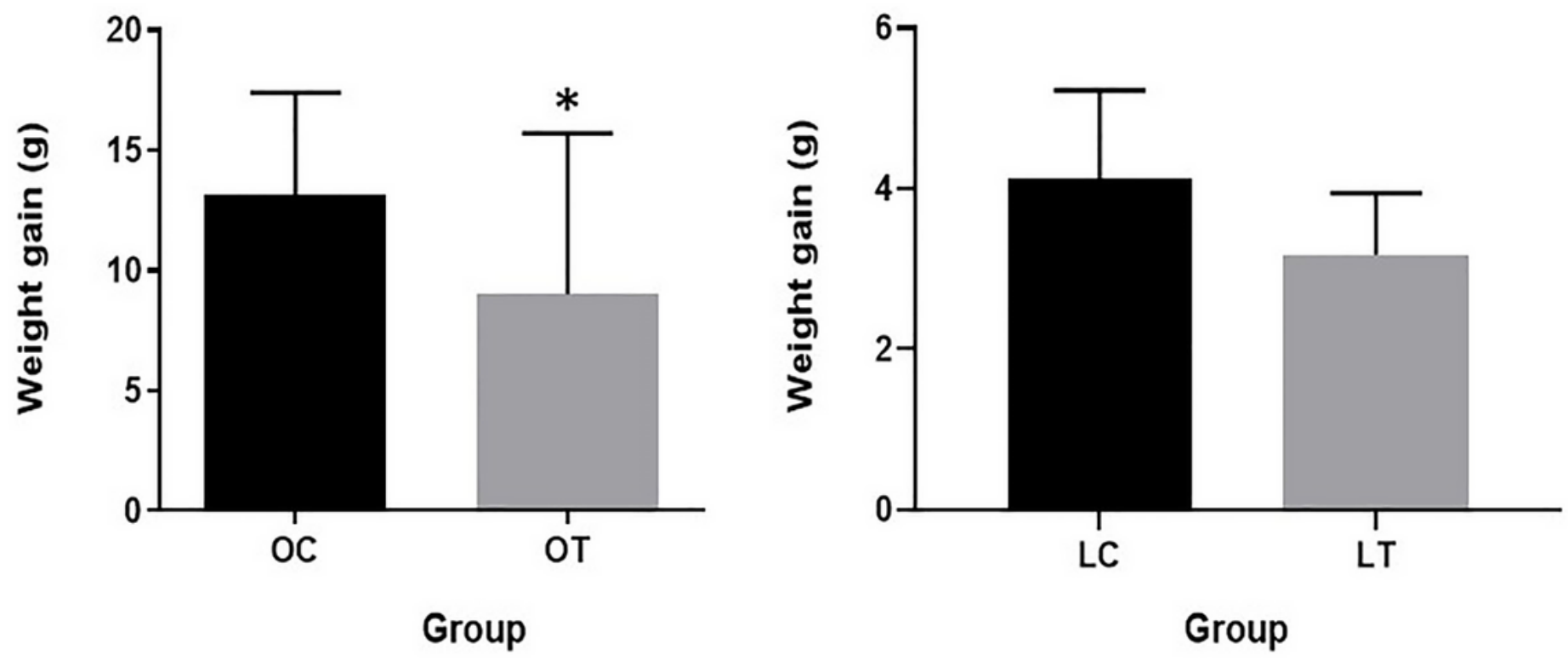

Figure 1 
The body weight gain after synbiotic supplementation of obese control (OC), obese treated (OT), lean control (LC) and lean treated (LT) animals.
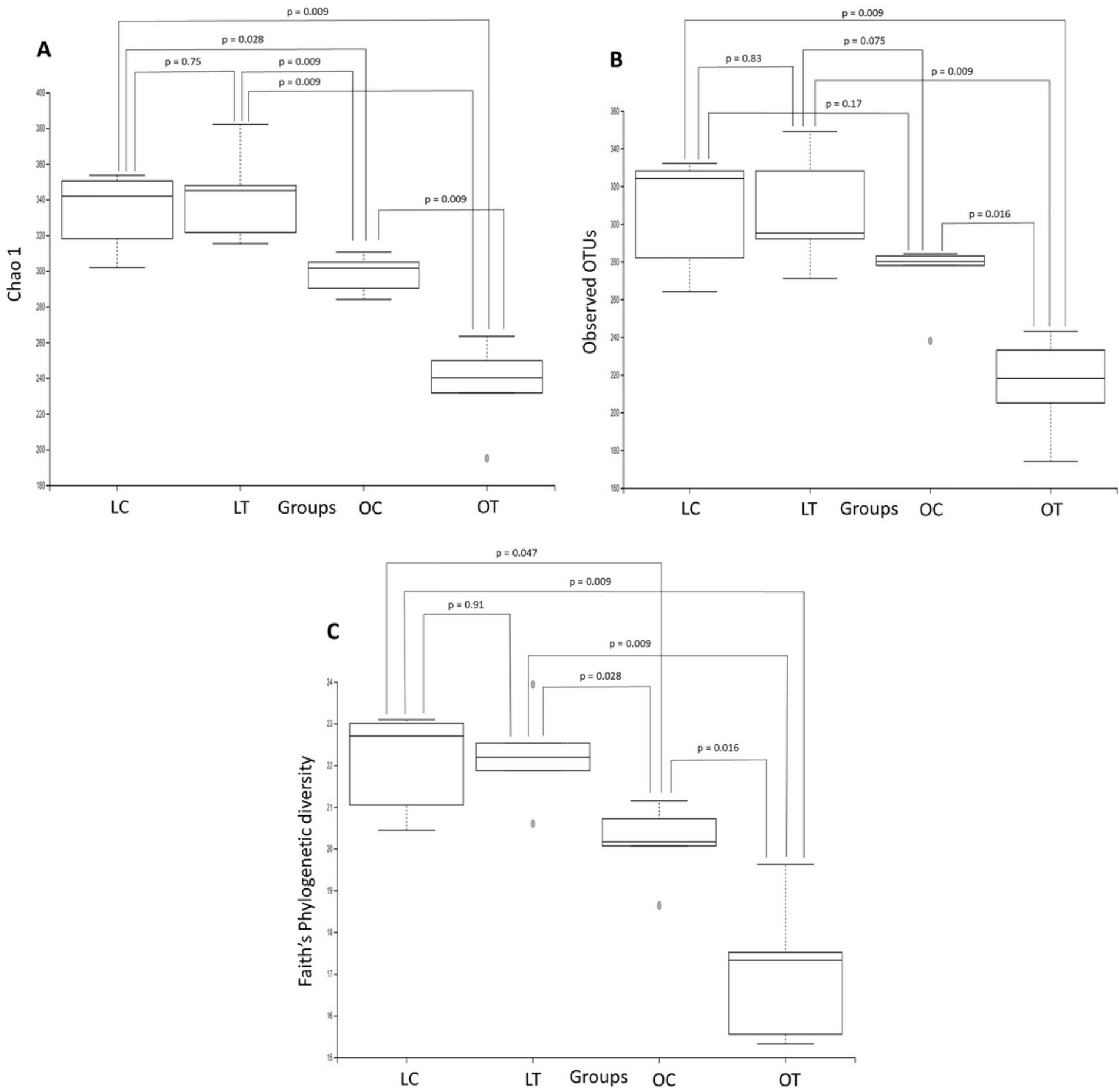

Figure 2

Comparison of the gut microbiota structures in lean control (LC), lean treated (LT), obese control (OC) and obese treated (OT) groups. The boxplots of Chao1 index (A), observed OTUs (B) and Faith's Phylogenetic (C) were constructed to evaluate microbiome diversity. 

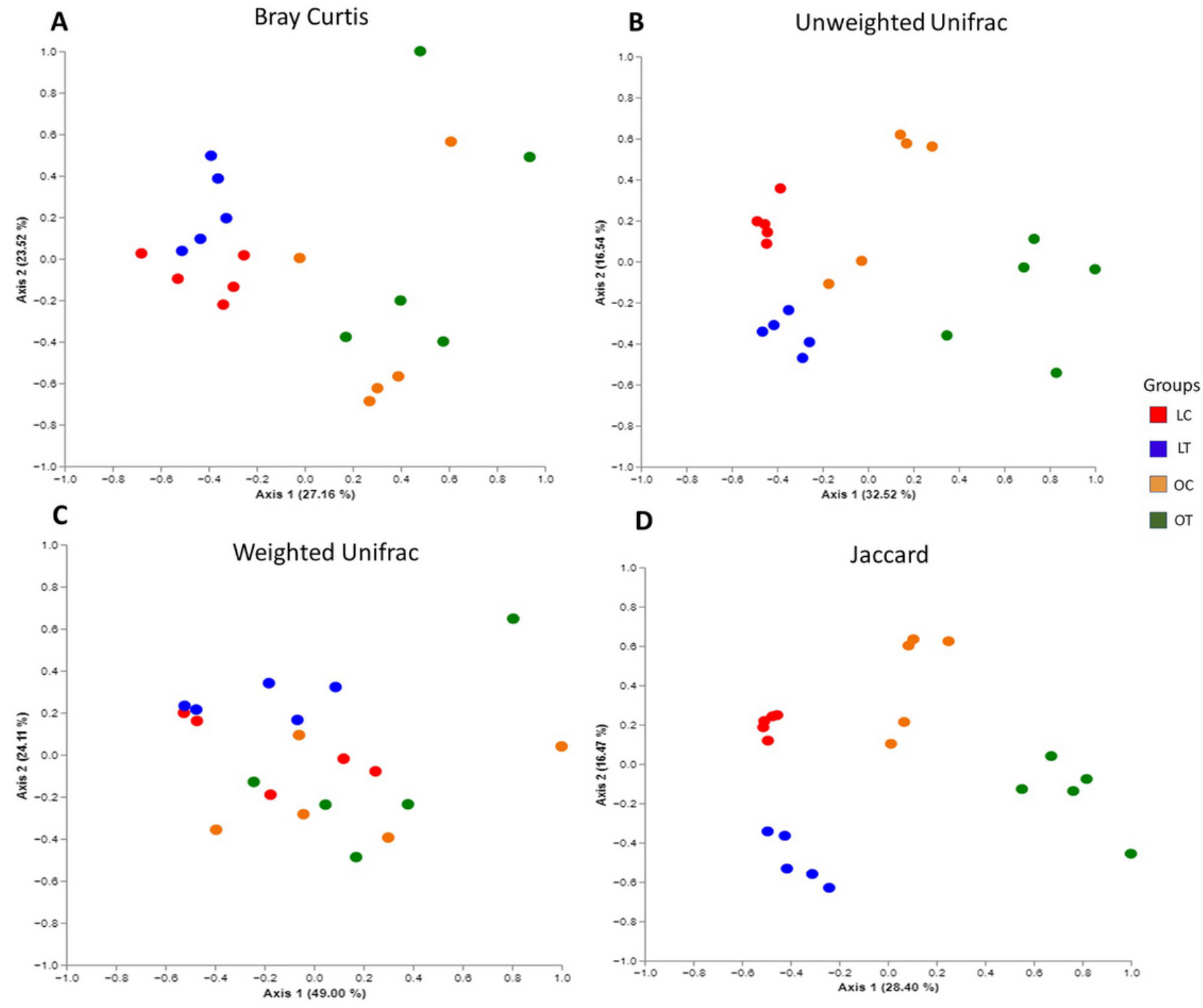

\section{Figure 3}

The composition changes of gut microbiota based on PCoA including Bray Curtis (A), Unweighted unifrac (B), weighted unifrac (C) and Jaccard (D) analysis for lean control (LC), lean treated (LT), obese control (OC) and obese treated (OT) groups. 


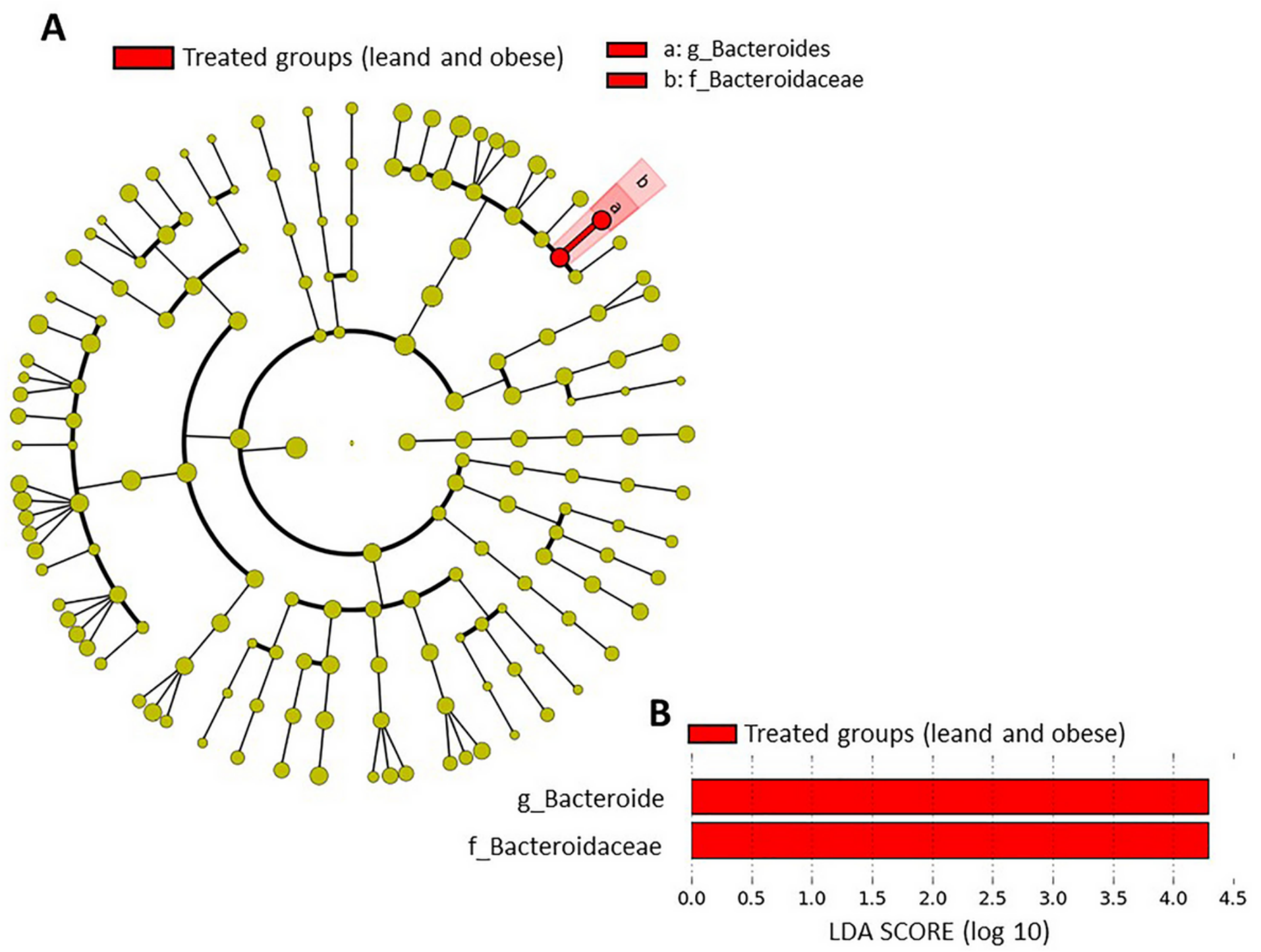

Figure 4

Taxonomic differences of fecal microbiota between groups. Taxa enriched in treated groups (lean and obese) have a positive score (red) (A). Only taxa meeting an LDA significant threshold $>2$ are shown (B). 


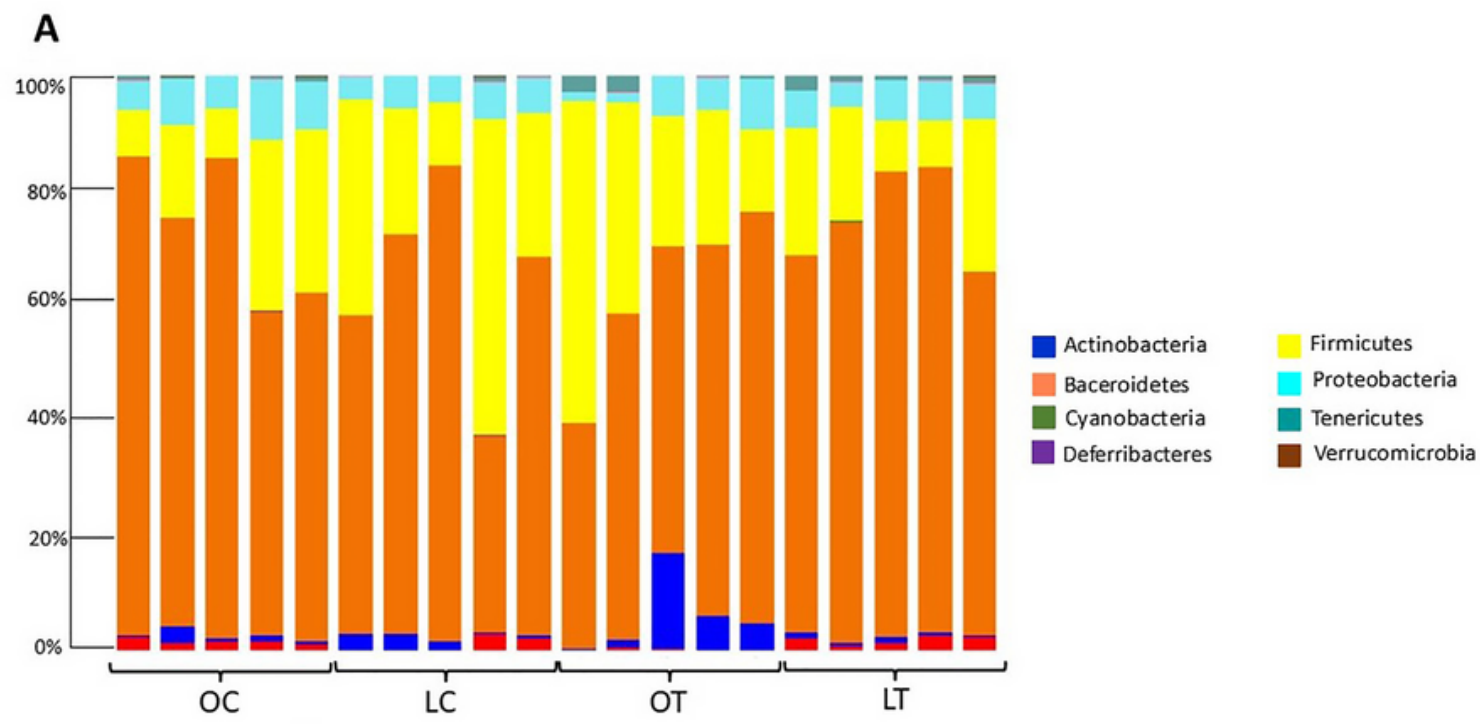

B

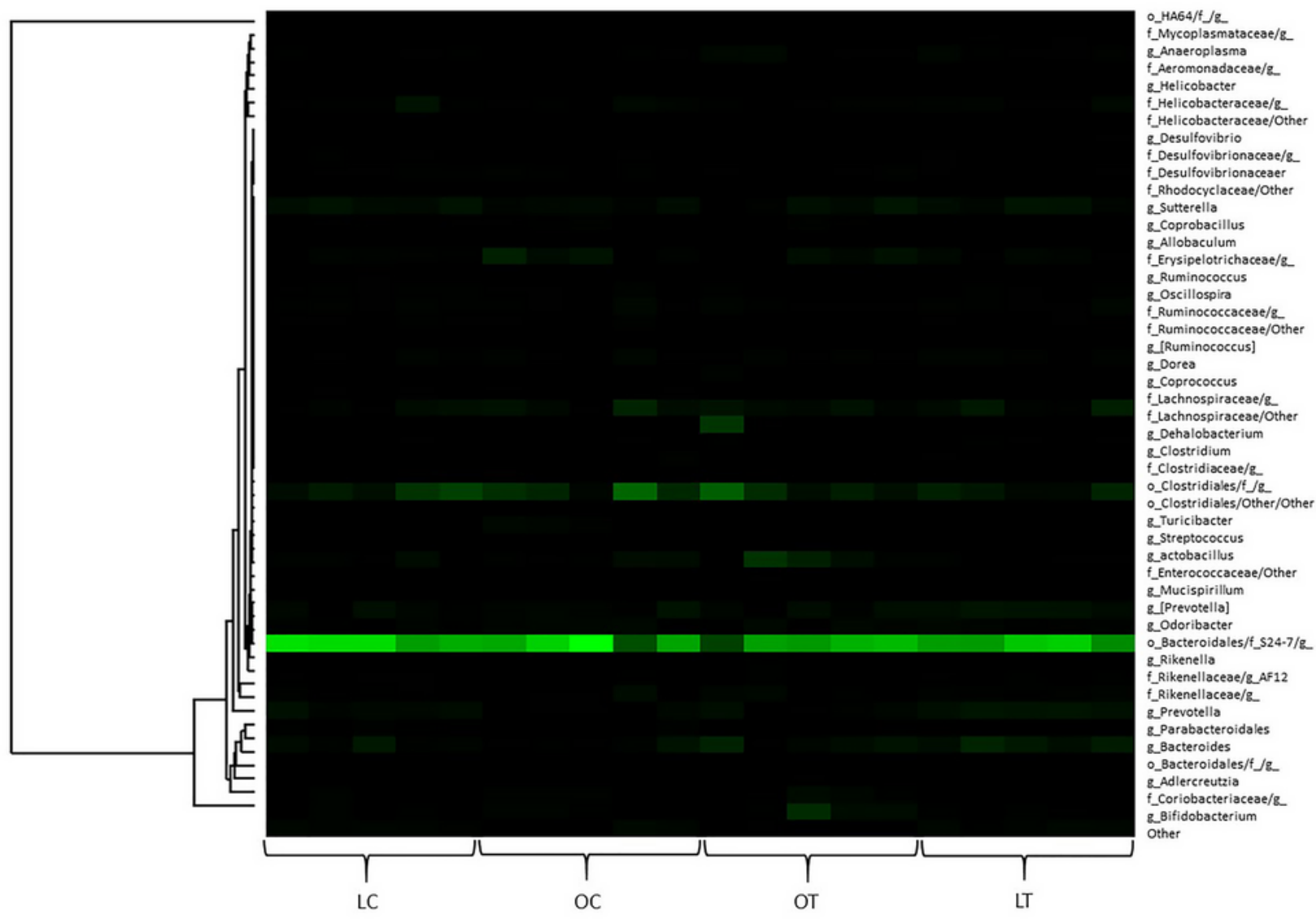

Figure 5

Relative abundance of bacteria represented by operational taxonomic unit (OTUs) in different sample storage conditions according to phyla (A). Heatmap of relative abundance at genus level (B). OC: Obese Control Group; LC: Lean Control Group; OT: Obese Treated Group; LT: Lean Treated Group. 

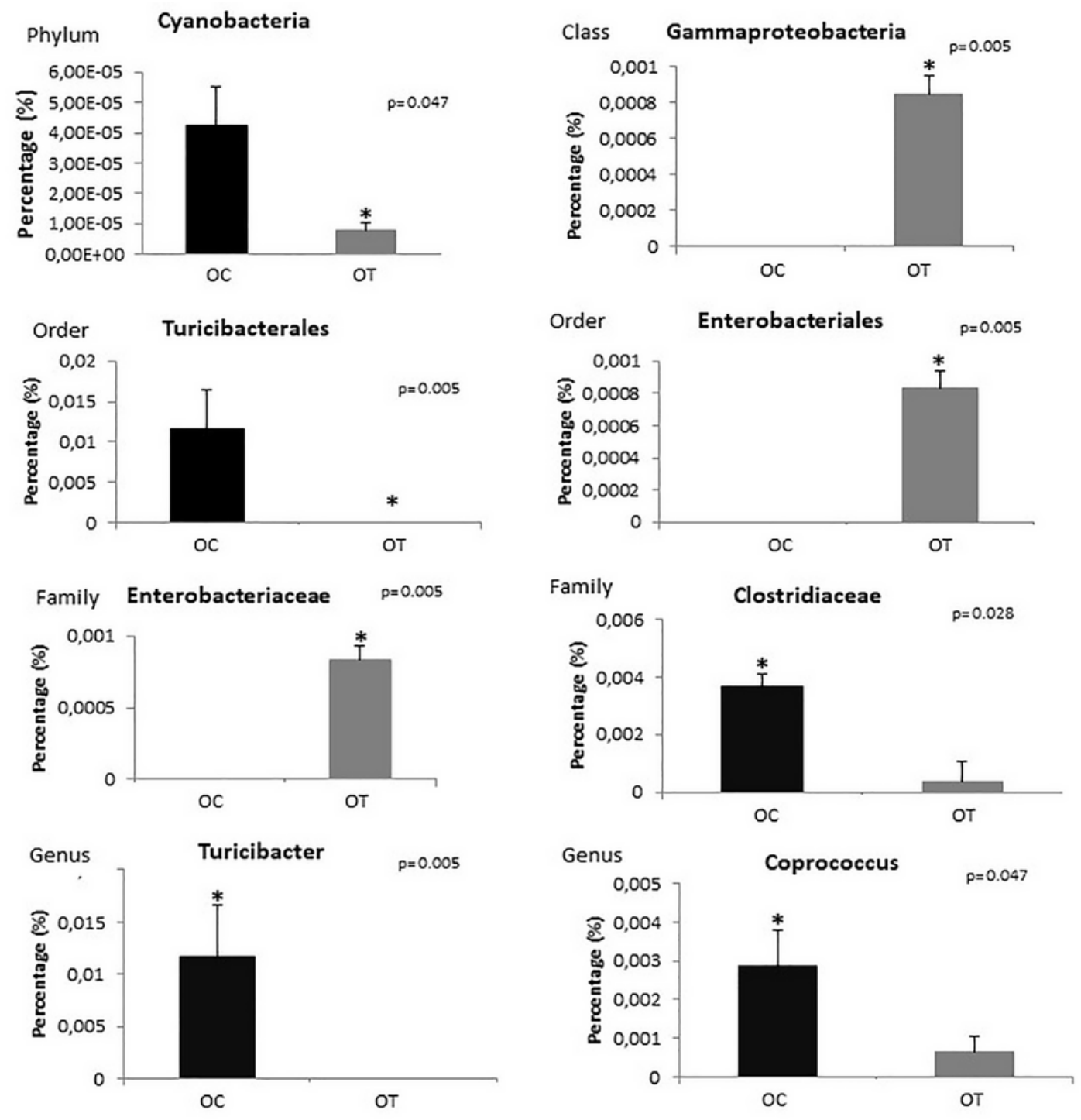

\section{Figure 6}

Analysis of the distribution of bacteria in obese control (OC) and obese treated (OT) groups according to phyla, class, order, family and genus. 


\section{Image not available with this version}

Figure 7

Analysis of the distribution of bacteria in lean control (LC) and lean treated (LT) groups according to phyla, order, family and genus.
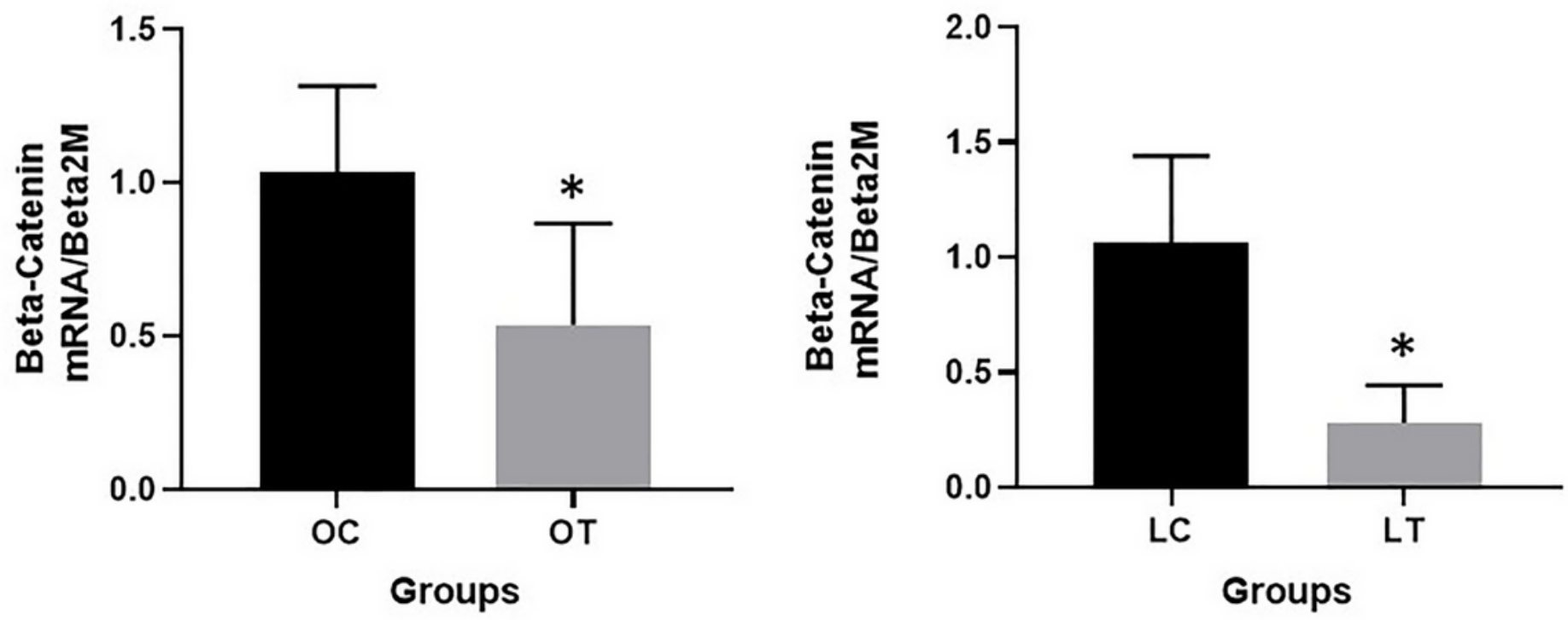

Figure 8

Beta-catenin expression in the gut of animals: obese control (OC), obese treated (OT), lean control (LC) and lean treated (LT) groups.

\section{Supplementary Files}

This is a list of supplementary files associated with this preprint. Click to download. 
- SupplementaryInformation.docx

- Additionalfile2.jpg 